\title{
Navigation for Human-Robot Interaction Tasks
}

\author{
Philipp Althaus*, Hiroshi Ishiguro ${ }^{\dagger}$, Takayuki Kanda ${ }^{\dagger}$, Takahiro Miyashita ${ }^{\dagger}$ and Henrik I. Christensen* \\ *Centre for Autonomous Systems, Numerical Analysis and Computer Science, \\ Royal Institute of Technology (KTH), S-10044 Stockholm, Sweden \\ [philipp, hic]@nada.kth.se \\ ${ }^{\dagger}$ ATR Intelligent Robotics and Communication Laboratories, 2-2-2 Hikaridai, \\ Keihanna Science City, Kyoto 619-0288, Japan \\ [ishiguro, kanda, miyasita]@atr.co.jp
}

\begin{abstract}
One major design goal in human-robot interaction is that the robots behave in an intelligent manner, preferably in a similar way as humans. This constraint must also be taken into consideration when the navigation system for the platform is developed. However, research in human-robot interaction is often restricted to other components of the system including gestures, manipulation, and speech. On the other hand, research for mobile robot navigation focuses primarily on the task of reaching a certain goal point in an environment. We believe that these two problems can not be treated separately for a personal robot that coexists with humans in the same surrounding. Persons move constantly while they are interacting with each other. Hence, also a robot should do that, which poses constraints on the navigation system. This type of navigation is the focus of this paper. Methods have been developed for a robot to join a group of people engaged in a conversation. Results show that the platform's moving patterns are very similar to the ones of the persons. Moreover, this dynamic interaction has been judged naturally be the test subjects, which greatly increases the perceived intelligence of the robot.
\end{abstract}

\section{INTRODUCTION}

Navigation is a necessary capability for mobile robots to move around in their surroundings. This issue is a widely studied research topic. A large number of strategies and systems have been developed for all kinds of environments. Most of these studies focus on reaching a certain goal point in a given environment while avoiding collisions on the way. A typical example is a robot for indoor environments fulfilling fetchand-carry type missions without human interaction during execution of the navigational task [1], [2]. However, in the research field of personal robotics [3] human-robot interaction is a major issue to be considered. The goal is to develop socially interactive robots [4] that "live" in people's homes and coexists with humans in their daily life. Also for these types of tasks, the movement of the platform is a basic problem that must be tackled. The constraints on the navigation system, however, are in some ways very different than for a fetchand-carry type missions.

A robot that has to reach a certain goal point in its environment uses, most often, some kind of map. This world model enables the system to create a plan and keep track of its execution. Hence, the state of the robot is mainly related to locations in its surrounding. In human-robot interaction, however, this state is more complex and a richer world model than a map of the environment is needed. The actions of the robot are dependent on the presence of humans and on their willingness to interact. Furthermore, the type and degree of interaction determines the subsequent behaviour. Also outcomes of a previous encounter can influence this interplay. For example, when a robot greets somebody, the response of this person determines the subsequent actions: shaking hands, engaging in a conversation, following the person to another room, and so forth. If this particular person is met again later, the interaction might be based on the first meeting. For example, the same questions should not be asked again.

Another new issue for navigation arises in interaction tasks, which concerns the actual control of the platform. A major constraint is that the robot's behaviour must look "natural". The movements have to be smooth and "human-like", since a personal robot is supposed to live, communicate and interact with people sharing the same environment. The quality of its movements influences strongly the perceived intelligence of the robotic system. As opposed to a delivery robot, which has to reach a certain destination with few constraints on the motion pattern. There, the only requirements are that the platform gets to the goal safely and in reasonable time.

These two problems, the world model for navigation and the control of the platform, have been tackled by extending an existing navigation system for indoor environments [1], [2]. This system is designed using a behaviour based approach [5]. The individual behaviours solve navigational subtasks, for example avoiding obstacles, following corridors, and passing doorways. The activation of these subtasks is governed by the robot's state, in other words its position in the environment. The world model reflecting these locations is a topological map. In the work presented in this paper, the topological map has been extended to a state diagram. This diagram incorporates both locations in the environment and events of an interaction task. Moreover, new behaviours were defined in order to execute a test scenario. This mission consists of: 1) entering a room, 2) approaching a group of people engaged in a discussion, 3) being part of this group by executing similar moving patterns than the persons, and 4) leaving the discussion and continuing navigation through the environment. Results 
show that the robot's behaviour is judged naturally and closely resembles the one of humans. This test scenario is a simplified task in order to verify the developed methods. Nevertheless, it is an initial step to studying navigation for human-robot interaction in particular, which we believe is necessary for developing intelligent personal robots.

In section II, the robotic platform and the interaction scenario tested in these studies are presented. The design and implementation of the actual system is described in section III and experimental results are presented in section IV. Finally, a summary and a discussion of avenues of future research are provided in section $\mathrm{V}$.

\section{THE EXPERIMENTAL SETUP}

An experimental setup was defined in order to demonstrate navigation for a human-robot interaction task. First, in section II-A, the robotic platform Robovie is described. An actual scenario, relevant for a robot sharing an environment with humans, is described in section II-B. In such a scenario, task constraints are usually given by psychological studies on human behaviour. These considerations are presented in section II-C.

\section{A. The Platform}

The platform used in these studies is a humanoid system on wheels called Robovie (Fig. 1). The robot has a height of $120 \mathrm{~cm}$ and its weight is about $40 \mathrm{~kg}$. The system is built on a Pioneer platform of $45 \mathrm{~cm}$ diameter with a two wheel differential drive. The robot has two arms with 4 degrees of freedom each. The head can be rotated around all three axis. Mounted on the head are two cameras that have also 2 degrees of freedom. There are various sensors mounted on the platform. The base has 16 ultrasonic sensors and encoders on the wheel for odometric information. Another 8 sonars are mounted on the upper body. Furthermore, the robot is equipped with 3 IR motion sensors on its shoulders, the two cameras on the head, and an omnidirectional camera on a sort of antenna. A number of touch sensors are distributed all over the body. In addition, two microphones and a loudspeaker provide a speech interface. The whole system is controlled by an onboard computer with a Pentium III processor running Linux.

Robovie was used in various studies on human-robot interaction [3], [6]. The system has modules for detecting moving objects and tracking faces. It is able to perform a number of gestures like greeting, waving, pointing, or hugging. Through moving its head it can give a realistic impression of attention. The speech interface allows some basic communication.

\section{B. The Scenario}

A robot sharing its environment with humans as an equal being must be able to engage in conversations. In order to do so, the system needs several abilities, which can be tested with the following scenario:

1) The robot enters a room and looks around for people.

2) If any humans are present, the platform approaches them.

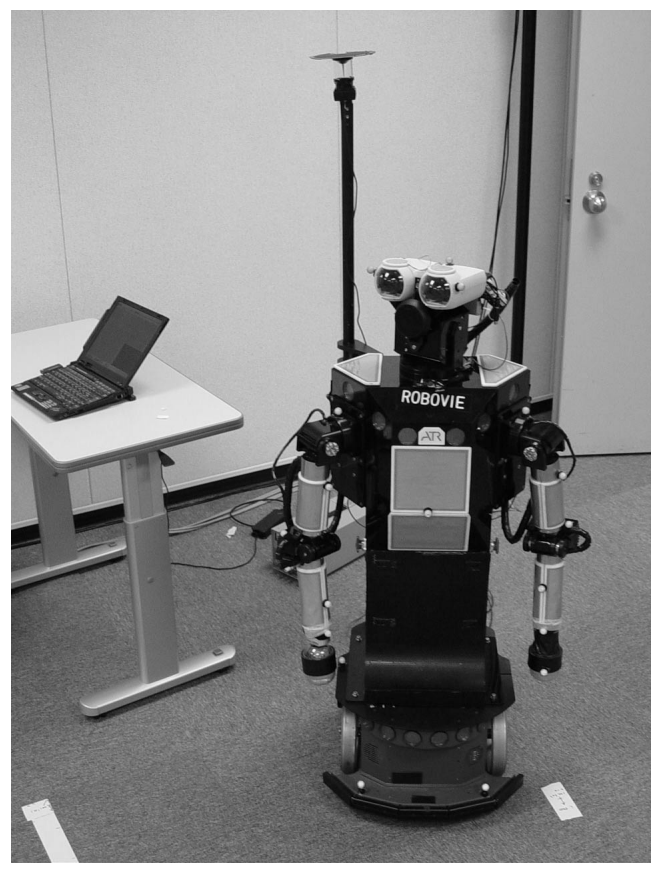

Fig. 1. The robotic system Robovie.

3) The robot stands next to the person(s) and initiates or joins a discussion.

4) When the discussion is over or the robot is asked to do something else, it leaves the person(s) towards the exit of the room.

5) The system resumes some old plan, responds to some new request, or wanders around in its environment.

Note that the emphasis of these studies is navigation. In the actual experiments (see section IV) the robot is in fact not really communicating. Keeping up a conversation over a reasonable time is very hard and a research field in itself. In other words, the platform is joining the group of people as a passive listener. This scenario is a minimal approach to demonstrate navigation in human-robot interaction including the following important issues:

- Detecting the presence of people.

- Moving in an appropriate fashion in relation to humans.

- Sequencing of different subtasks.

- Keeping track of position and plan execution.

\section{Psychological Considerations}

The design of autonomous systems for human-robot interaction is highly influenced by psychological studies. There exists a great amount of results from this area describing the way how humans interact with each other. It is the aim for a personal robot to follow the same principles when acting in a human environment. This enables the acceptance of a robotic platform as an equal being on the one hand. On the other hand, it increases the perceived intelligence of the system [7]. Furthermore, psychological methods in the form of questioning human subjects about their impression of a robot, 
are a valuable method to evaluate and measure the quality of an interacting system [8].

This chapter deals with the navigation aspects of an interaction task. Hence, studies about people's overall movements and their position relative to each other are taken into account. Humans try to keep a certain distance to others, which changes with the type of relationship they have among them [9]. In addition, multiple humans keep a formation during conversation. Results of extensive studies on this topic are presented in [10]. The main points being that certain formations among people are maintained, while they are interacting. Although individual people often are moving due to different reasons, the other persons are compensating for this by also moving in order to maintain the formation. In a group conversation, this formation usually has the form of a ring, where all persons keep equal distance to their neighbours. Of course, there are many variations and exceptions of these general rules depending on the relation among the persons and the state of the conversation. However, the main principle described above is rather dominant and will define the constraints for the design of the control system.

\section{IMPLEMENTATION}

The implementation of the scenario described above is described in this section. An existing navigation system for delivery missions in domestic environments [1], [2] was extended to incorporate interaction tasks. This system is based on the behaviour based design methodology. Furthermore, it makes use of a world model in the form of a topological map of the environment. This map and its extension to a state diagram are introduced in section III-A. Then the control of the platform is presented in section III-B.

\section{A. Topological Map and State Diagram}

The topological map allows the robot to make decisions about which navigational subtasks to initiate. The main structure contains qualitative information about the large-scale connectivity of the environment, which is modelled as a collection of rooms, corridors, and doorways. This information is reflected in a graph structure containing nodes and edges that connect these nodes. The nodes stand for important places in the environment and locations where a change in the navigational strategy occurs. Hence, there has to be one in front of each door, at each corridor crossing and at other places of interest (for example, goal locations and charging station). Each node has a location in a fixed coordinate system. The edges that connect these nodes can be of three different types: "room", "corridor", or "door". Fig. 2 shows the map of the laboratories at ATR. Due to the limited amount and simplicity of information in this map, it is a matter of minutes to construct a new one for a previously unknown domestic environment. Nevertheless, it has been shown that this world model is enough to allow navigation between two arbitrary points in a cluttered large-scale setting [1], [2].

A state diagram is defined preserving the graph structure of the topological map. Fig. 3 shows this diagram for the

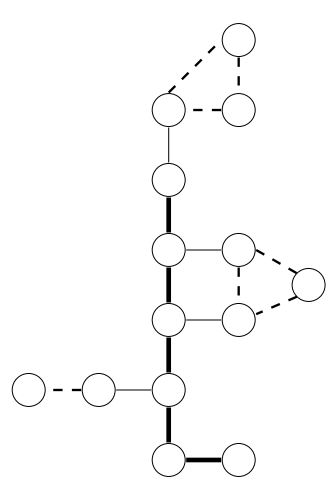

Fig. 2. The topological map of the laboratories at ATR. The circles depict nodes, which have an exact location in a coordinate system. Edges are of three different types: corridor (thick line), room (dashed line), and door (thin line). Additional nodes for goal points and starting positions can be added arbitrarily.

scenario described in section II-B. Now, nodes reflect both locations in the environment and events in the interaction task. Since events can not occur in any arbitrary order, the edges have to be directed (as opposed to the topological map). These edges are still of a certain type associated with a subtask. In addition to "room", "corridor", and "door", they can be of types "approach" and "join" as well. These types determine the activation of behaviours introduced later in this section. The edge of type "approach" corresponds to the activation of a behaviour APPROACH HUMAN. The edge of type "join" corresponds to the subtask of joining the group of people and maintaining the formation relative to them. In other words, a behaviour KEEP DISTANCE is active.

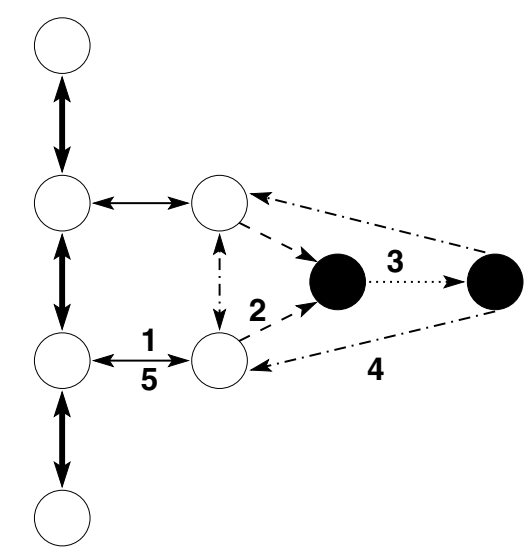

Fig. 3. The state diagram representing a corridor on the left with two doors into a large room, where the interaction scenario described in section II-B takes place. White nodes depict locations and black nodes correspond to events. Edges can be of five different types: corridor (thick line), door (this line), room (dashed-dotted line), approach (dashed line), or join (dotted line). In the experiment described in section IV, the system follows the edges according to the numbers: 1) entering the room, 2) approaching the group of people, 3) maintaining a formation with the persons, 4) getting back to the door, and 5) leaving the room again.

Note that, in this diagram, nodes correspond to events and edges to tasks in order to preserve the topological map structure. This is an obvious difference to finite state acceptor 
diagrams [11] and discrete event systems [12], where the role of edges and nodes is reversed. Furthermore, the diagram as depicted in Fig. 3 is not a perfect solution yet. In a larger environment, the scenario of joining a group conversation could happen at many different places. Which means that the nodes and edges reflecting this interaction would have to be added to the map many times. A solution to this redundancy could be to define a third dimension for event nodes. Then, all location nodes could, in theory, have a connection to the same node in this additional dimension.

\section{B. Control}

The control scheme is designed in the framework of behaviour based robotics using the dynamical systems approach introduced in [13], [14]. To provide the functionality of joining a group of people and engaging in a conversation two additional behaviours were defined: APPROACH HUMAN and KEEP DISTANCE. These behaviours are integrated into the existing control system presented in [1], [2]. At any time, these behaviours define the desired heading direction and velocity of the robot. Here, only the basic concepts are explained. For a detailed discussion including stability analysis of the controllers we refer to [15]. The implementation is described below following the individual steps as depicted in Fig. 3

1) Entering the room: The robot performs the navigational task of reaching a goal point in a room just behind the door. This is performed by a behaviour DOOR PASSING as described in [1], [2].

2) Approaching the group of people: After the robot has entered the room, a person tracking module is initiated. This module looks for a person using a combination of data from different sensors: the omnidirectional camera, one of the cameras on the robot's head, the sonar sensors and infrared sensors mounted on the shoulders of the robot. The output of this module specifies the direction $\psi_{\text {human }}$ of the human. With help of the sonar sensors the distance $d_{\text {human }}$ to this person can be determined.

The behaviour APPROACH HUMAN aligns the robot's heading direction with the direction $\psi_{\text {human }}$ of the detected person. The desired speed is dependent on the distance $d_{\text {human }}$ to the people that have to be approached. In the same way as when a human is approaching a group of people, the platform should slow down when getting closer. Furthermore, the speed ought to be bounded by some maximum value $v_{\max }$ in order not to scare anybody. Hence, the commanded speed is defined as follows

$$
v=\min \left(k_{\text {human }} d_{\text {human }}, v_{\max }\right)
$$

where $k_{\text {human }}>0$ is a constant.

3) Maintaining a formation with the persons: After the robot has reached the group of people, individual persons must be detected in order to be able to keep a certain formation. This is done using sonar data for a rather simple geometric representation of people. The 30 most recent sonar readings are considered. Persons are reconstructed from these detected echos in ascending order of their distance to the robot. The echo closest to the robot defines the first person whose orientation in the robot frame is given by the axis of the sensor that received the echo. A new person is recorded for every subsequent echo whose orientation differs by an angle of at least $45^{\circ}$ from any previously identified person. New persons are added in an incremental fashion until the sonar buffer contains no further echos. This defines a direction $\psi_{j}$ and a distance $d_{j}$ for each person $j$.

In the case of one person $j$ present the behaviour KEEP DISTANCE turns the robot towards it $\left(\psi_{j}\right)$. Furthermore, it has to make the platform back off when the person gets closer than the desired predefined interaction distance $D_{\text {inter }}$. If the distance, however, becomes greater the robot should approach. In order to avoid abrupt changes in movement, these speeds are also regulated by the distance $d_{j}$ to that person. Thus the desired speed can be expressed in the following form.

$$
v_{j}=k_{\text {dist }}\left(d_{j}-D_{\text {inter }}\right)
$$

with a constant $k_{\text {dist }}>0$ and an interaction distance defined by $D_{\text {inter }}$.

In the case of several persons present the robot should face the middle of the group (see section II-C). This can be achieved by defining the robot's heading direction $\psi$ through averaging the directions $\psi_{j}$ of each individual person.

$$
\psi=\frac{1}{N} \sum_{j} \psi_{j} e^{-c\left(d_{j}-D_{\text {inter }}\right)}
$$

$N$ is the amount of people detected and $c>0$ defines a decay constant. The distance dependent decay term has the purpose of "repairing" a formation. If one person in the group steps closer to the robot $\left(d_{j}<D_{\text {inter }}\right)$, its contribution becomes stronger than the others. This ensures that the platform is turning towards this person, while backing off. Subsequently, when the distance increases again, it is aligning itself with the center of the formation. The desired speed of the robot is defined in the same fashion. The resulting speed is a weighted average of the values $v_{j}$ defined in (2) for each detected person.

$$
v=\frac{1}{N} \sum_{j} v_{j} e^{-c\left(d_{j}-D_{i n t e r}\right)}
$$

Also here, the distance dependent term weighs the contributions from closeby people stronger than the ones from people further away. This always ensures that the platform is avoiding persons that are standing too close. For more details and illustration on the behaviour emerging from these definitions see the results in section IV.

4) Getting back to the door: In the present implementation, the end of the interaction is triggered by a timer. In other words after a certain amount of time the robot leaves the group of people again. However, with a real communication robot the actual end of a discussion could be detected, which leads to more natural behaviour. Subsequently, the navigation behaviours for delivery missions [1], [2] take control of the platform. Hence, the robot is driving back to the door and executing some arbitrary mission with the help of the topological map. 


\section{RESUlts}

The scenario described in section II-B was tested. The robot entered a room, detected a group of three humans engaged in a conversation, and joined this group. While talking to each other, the persons were moving slightly. Further, one of these persons was leaving the group at some point. Later, this person was coming back and joining the group again. During all these movements of the people, the robot had to adjust its own position to remain in a formation as described in section II-C. Moreover, the robot turned its head towards the person closest to it in order to give an impression of listening to the conversation. Finally, the robot was leaving the group and heading for the door where it entered the room in the first place. Robovie was not engaged in the conversation and acted as a passive listener. Therefore, leaving the group is not triggered by the end of the conversation. The robot simply returns to its old task after 2 minutes of listening. The path through the state diagram for this task can be seen in Fig. 3.

The three subjects used for the experiment have never seen the robot before. This was an important condition, because they must not know about the platform's moving patterns and automatically adjust to it. The main constraints on the navigation are that the robot moves naturally in a similar way as humans do. The subjects where questioned about their impressions after the experiment. They judged the moving patterns of the robot as natural and had the impression that Robovie was listening to their conversation.

A video was taken of the experiment. However, these kind of results are very difficult to present on paper. An attempt to give an impression of the experiment is done by displaying trajectories. The room where the interaction took place is equipped with a system for tracking optical beacons with a large number of cameras. Such beacons were placed on the subjects and the robot in order to track their position during the experiment. The movements of the robot and the subjects during a conversation with a constant amount of people are really small and hard to draw and interpret. However, during major events, for example when a person is leaving, the robot has to move greater distances to remain in formation with the humans. The trajectories during some of these major events are displayed and discussed below.

Figs. 4 to 7 show the trajectories of the robot (dashed line) and the subjects (solid lines) for four short sequences during the interaction task. The units are measured in meters. The circles indicate the approximate size of the robot and the persons. The dashed circles indicate the position at the beginning of the sequence. The solid ones show the final positions when the pictures below the plots were taken.

Fig. 4 shows the trajectories just after the robot has entered the door and is approaching the group of people. Before the robot got there the persons were arranged in a regular triangle (dashed circles). As soon as the robot came close, they rearranged their positions to open up space for the platform. Robovie positioned itself such that the four have about equal distances to each other.
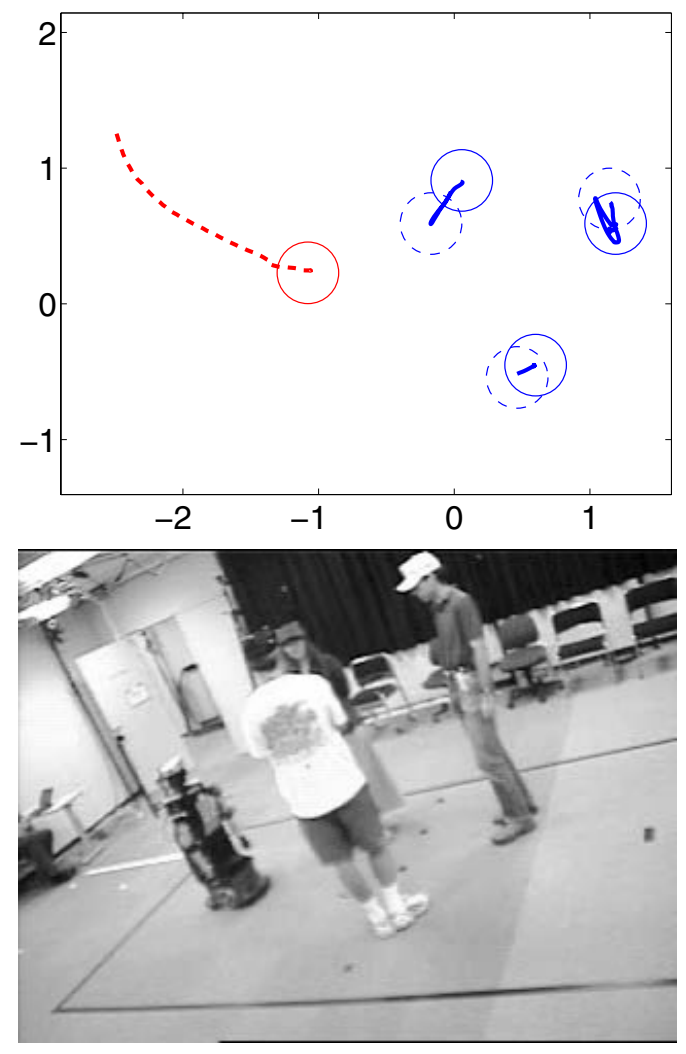

Fig. 4. The robot approaches the group of people.
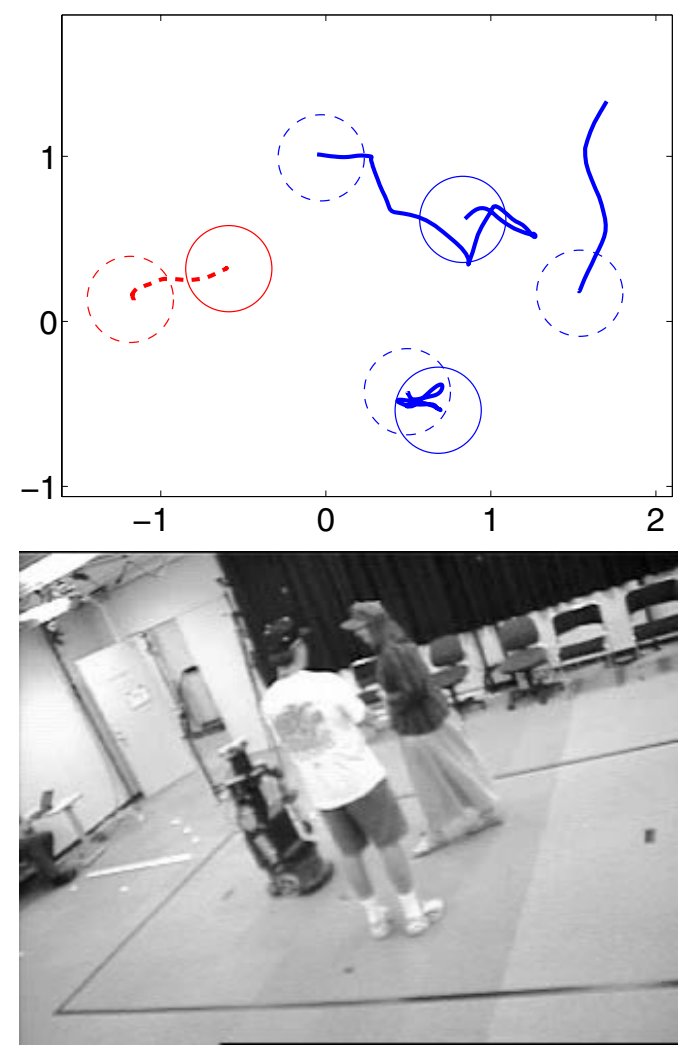

Fig. 5. The person on the right hand side is leaving the formation. 

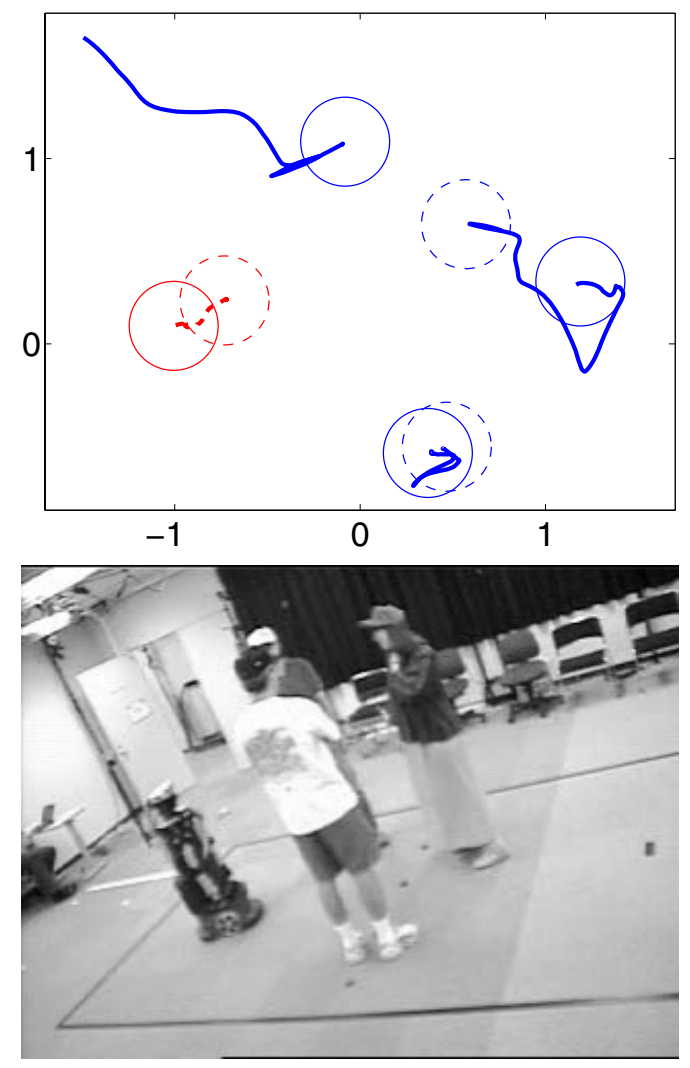

Fig. 6. The third person is returning and approaching the group.

In Fig. 5 maintaining formation after a person was leaving can be observed. At the beginning of the sequence the four stood at about equal distances to each other (dashed circles). Then, the person on the right hand side was leaving. The other two and Robovie were moving in such a way that the new formation constitutes a rather regular triangle (solid lines).

In Fig. 6, it can be observed how the subjects were moving when the third person joined the group again. At first, the two persons and the robot were at similar positions as at the end of the sequence of Fig. 6. Then the third person was approaching from the top left. Robovie and the person on the right are opening up space, such that the four of them constitute a similar formation as in the beginning of the sequence of Fig. 6.

Fig. 7 displays the end of the interaction task. The dashed circles show the individuals in regular formation. Then after 2 minutes of interaction, the end of the task was triggered and the robot left the group in the direction from where it came from.

\section{DisCUSSION}

A basic scenario for testing navigation in human-robot interaction tasks has been defined. Two behaviours have been designed, which facilitate the system to approach a group of people and to maintain a certain formation while interacting. Experiments showed that the robot is able to reposition itself when these formations change due to different events. Furthermore, the way the robot moves and finds its position in the

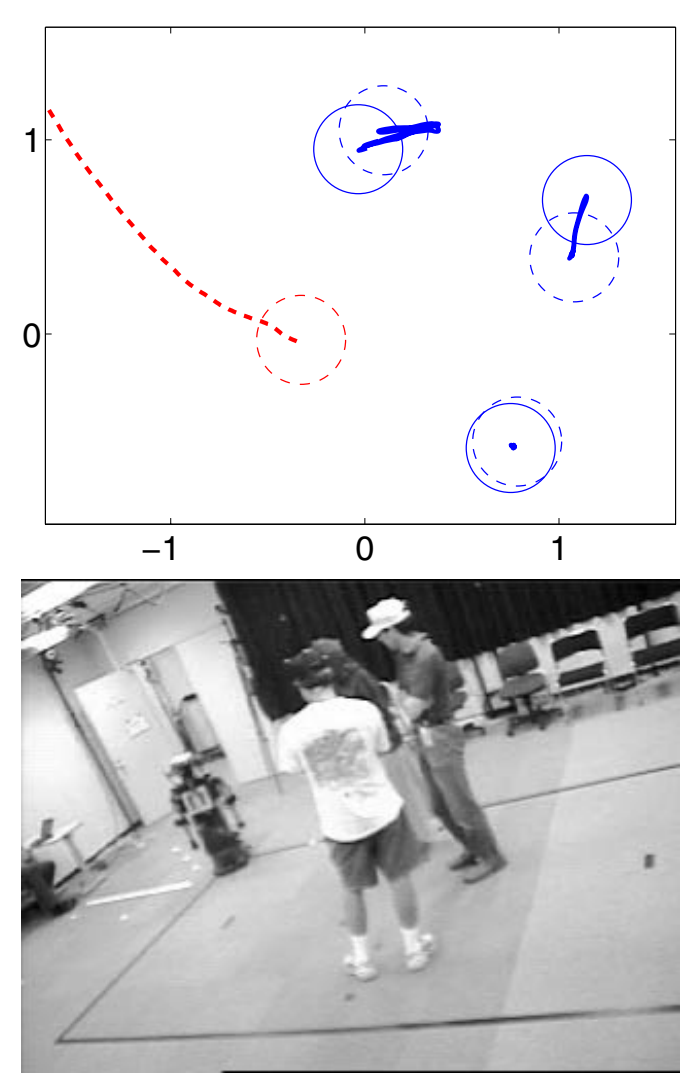

Fig. 7. The robot leaves the group of people.

group has been judged naturally by the test subjects.

Human-robot interaction poses different constraints on navigation than delivery missions, which are the context of most navigation studies. However, in this paper the following could be shown: 1) The dynamical systems approach is also suited for behaviour design in these types of tasks. 2) The world model (topological map) could easily be extended in order to contain information relevant for interaction. In essence, this shows that the methodologies used in earlier work [1], [2] are not restricted to fetch-and-carry type mission. They can easily be extended to form the basis of more general navigation systems.

There are a number of other robotic systems that move around in spaces shared with humans and that engage in some form of interaction (see [4] for a survey). One type of systems that attract a great deal of attraction and operate in crowded spaces are museum tour guides. Rhino in the "Deutsche $\mathrm{Mu}$ seum Bonn" [16], its successor Minerva in the "Smithsonian's National Museum of American History" in Washington [17], and Robox at the "Swiss National Exhibition Expo02" [18] are the most popular examples. All these systems possess the ability to interact with humans. However, this interaction is based on speech, gestures, and other visual cues. Navigation is not considered in the context of an interactive purpose. In Robox, people are treated as dynamic obstacles only, which are fed to an obstacle avoidance module [19]. In Rhino and Minerva, humans are detected by cameras in order to initiate 
interaction tasks [20]. These tasks are restricted to the upper body of the robot and do not influence the navigation system.

Only recently, some research has been devoted to the role of navigation for socially interactive robots. Simply turning towards persons of interest allows the robot to express some personality [21], [22]. Furthermore, some first considerations of human-robot formations for cooperative manipulation are done in [23]. Moreover, the personal spaces of humans similar to the psychological arguments in section II-C are disussed in [24]. There, these constraints are considered when implementing a robotic system for standing in a line to get a coffee. The work presented in this paper is another initial example of incorporating navigation into the interaction loop. We believe that this is necessary and will be seen in personal robotic systems in the future.

The design and results presented in this paper are clearly some simplified initial studies. Human formations are, in general, more complex than discussed here. For example, the distance to other people is dependent on the personal relations among them [9]. Also the type of interactive means used (gestures or speech) influence the formations among the participants. This information could be incorporated into a system by using modules for recognising individual humans and interpreting their gestures [25]. This system would also facilitate to notice differences between humans and objects like chairs. This again will lead to more advanced formation control than in the present implementation.

\section{ACKNOWLEDGMENT}

Thanks to Daniel Eaton for his help with the Robovie software. This research has been sponsored by ATR International Japan and the Swedish Foundation for Strategic Research. Moreover, funding from the Sweden-Japan Foundation Stockholm allowed the first author to spend four months at ATR in Japan. The support is gratefully acknowledged.

\section{REFERENCES}

[1] P. Althaus and H. I. Christensen, "Smooth task switching through behaviour competition," Robotics and Autonomous Systems, vol. 44, no. 3-4, pp. 241-249, 2003.

[2] - "Behavior coordination in structured environments," Advanced Robotics, vol. 17, no. 7, pp. 657-674, 2003.

[3] H. Ishiguro, T. Ono, M. Imai, and T. Kanda, "Development of an interactive humanoid robot "Robovie" - An interdisciplinary aproach," in Robotics Research, R. A. Jarvis and A. Zelinsky, Eds. Berlin: Springer, 2003, pp. 179-191.

[4] T. Fong, I. Nourbakhsh, and K. Dautenhahn, "A survey of socially interactive robots," Robotics and Autonomous Systems, vol. 42, no. 3-4, pp. 143-166, 2003.

[5] R. C. Arkin, Behavior-Based Robotics. Cambridge, MA: MIT Press, 1998.

[6] T. Kanda, H. Ishiguro, T. Ono, M. Imai, and R. Nakatsu, "Development and evaluation of an interactive humanoid robot "Robovie"," in Proceedings of the IEEE International Conference on Robotics and Automation, 2002, pp. 1848-1855.
[7] T. Kanda, H. Ishiguro, M. Imai, T. Ono, and K. Mase, "A constructive approach for developing interactive humanoid robots," in Proceedings of the IEEE/RSJ International Conference on Intelligent Robots and Systems, 2002, pp. 1265-1270.

[8] T. Kanda, H. Ishiguro, and T. Ishida, "Psychological analysis on humanrobot interaction," in Proceedings of the IEEE International Conference on Robotics and Automation, 2001, pp. 4166-4173.

[9] E. T. Hall, The Hidden Dimension. Garden City, NY: Doubleday, 1966.

[10] A. Kendon, Conducting Interaction. Cambridge University Press, 1990 ch. 7: Spatial organization in social encounters: the F-formation system, pp. 209-237.

[11] R. C. Arkin and D. MacKenzie, "Temporal coordination of perceptual algorithms for mobile robot navigation," IEEE Transactions on Robotics and Automation, vol. 10, no. 3, pp. 276-286, 1994.

[12] J. Kosecká and R. Bajcsy, "Discrete event systems for autonomous mobile agents," Robotics and Autonomous Systems, vol. 12, no. 3-4, pp. 187-198, 1994.

[13] G. Schöner and M. Dose, "A dynamical systems approach to task-level system integration used to plan and control autonomous vehicle motion," Robotics and Autonomous Systems, vol. 10, no. 4, pp. 253-267, 1992.

[14] G. Schöner, M. Dose, and C. Engels, "Dynamics of behavior: theory and applications for autonomous robot architectures," Robotics and Autonomous Systems, vol. 16, no. 2-4, pp. 213-245, 1995.

[15] P. Althaus, "Indoor navigation for mobile robots: Control and representations," Ph.D. dissertation, Royal Institute of Technology (KTH), Stockholm, S, 2003.

[16] W. Burgard, A. B. Cremers, D. Fox, D. Hänel, G. Lakemeyer, D. Schulz, W. Steiner, and S. Thrun, "Experiences with an interactive museum tourguide robot," Artificial Intelligence, vol. 114, no. 1-2, pp. 3-55, 1999.

[17] S. Thrun, M. Bennewitz, W. Burgard, A. B. Cremers, F. Dellaert, D. Fox, D. Hähnel, C. Rosenberg, N. Roy, J. Schulte, and D. Schulz, "MINERVA: A second-generation museum tour-guide robot," in Proceedings of the IEEE International Conference on Robotics and Automation, 1999, pp. 1999-2005.

[18] R. Siegwart, K. O. Arras, S. Bouabdallah, D. Burnier, G. Froidevaux, X. Greppin, B. Jensen, A. Lorotte, L. Mayor, M. Meisser, R. Philippsen, R. Piguet, G. Ramel, G. Terrien, and N. Tomatis, "Robox at Expo.02: A large-scale installation of personal robots," Robotics and Autonomous Systems, vol. 42, no. 3-4, pp. 203-222, 2003.

[19] R. Philippsen and R. Siegwart, "Smooth and efficient obstacle avoidance for a tour guide robot," in Proceedings of the IEEE International Conference on Robotics and Automation, 2003, pp. 446-451.

[20] J. Schulte, C. Rosenberg, and S. Thrun, "Spontaneous, short-term interaction with mobile robots," in Proceedings of the IEEE International Conference on Robotics and Automation, 1999, pp. 658-663.

[21] A. Bruce, I. Nourbakhsh, and R. Simmons, "The role of expressiveness and attention in human-robot interaction," in Proceedings of the IEEE International Conference on Robotics and Automation, 2002, pp. 41384142 .

[22] H. G. Okuno, K. Nakadai, and H. Kitano, "Realizing personality in audio-visually triggered non-verbal behaviors," in Proceedings of the IEEE International Conference on Robotics and Automation, 2003, pp. 392-397.

[23] J. Tan, N. Xi, A. Goradia, and W. Sheng, "Coordination of human and mobile manipulator formation in a perceptive reference frame," in Proceedings of the IEEE International Conference on Robotics and Automation, 2003, pp. 374-379.

[24] Y. Nakauchi and R. Simmons, "A social robot that stands in line," in Proceedings of the IEEE/RSJ International Conference on Intelligent Robots and Systems, 2000, pp. 357-364.

[25] M. Furukawa, Y. Kanbara, T. Minato, and H. Ishiguro, "Human behavior interpretation system based on view and motion-based aspect models," in Proceedings of the IEEE International Conference on Robotics and Automation, 2003, pp. 4160-4165. 